\title{
Radial Distribution System Reconfiguration in the Presence of Distributed Generators
}

\author{
Xingquan $\mathrm{Ji}^{1, \text { a }}$, Qi Liu ${ }^{1}$, GuoZheng $\mathrm{Han}^{1}$, Zhipeng $\mathrm{Liu}^{2}$ and Qingxue Liu ${ }^{1}$ \\ ${ }^{1}$ Shandong University of Science and Technology, Qingdao 266510, China; \\ ${ }^{2}$ Dispatching and Control Center of Hainan Power Grid Corporation, Haikou 570203, China. \\ axqji@163.com
}

Keywords: distribution system reconfiguration; distributed generation; genetic algorithm.

\begin{abstract}
A distribution network reconfiguration model with wind power generators is proposed, and an improved genetic algorithm is applied to minimize active power loss. Forward and backward sweep approach is adopted to calculate the power flow. In addition, the strategy of searching random spanning trees is used to ensure the radial structure of network in the process of reconfiguration. Simulation results show that the proposed method can reduce the power loss and improve the voltage quality of distribution system.
\end{abstract}

\section{Introduction}

As an effective measure, distribution system reconfiguration (DSR) is often used as a means to improve the system security or reduce the power loss of network. The common objective functions of distribution network reconfiguration model includes: active power loss, voltage level, load balancing, and system reliability.

The DSR problem with DGs incorporated has been recently addressed in different perspectives. Based on the information and functionality of remote controlled equipment, a methodology for real-time reconfiguration of distribution network considering DG was developed in [1]. In [2], DG placement was considered while solve the DSR problem using sensitivity analysis. In [3], a multi-objective framework was proposed which considering power losses, DG cost, greenhouse gas emissions, and voltage stability.

DSR is usually formulated as a complex combinatorial, nonlinear constrained optimization problem which is difficult to get the exact solution. Many kinds of algorithms are proposed in the past. Due to the advantages of global searching and inherently parallel, meta-heuristic algorithms, such as genetic algorithm (GA) [4], tabu search algorithm (TSA) [5], and particle swarm optimization (PSO) [6] have been successfully applied in DSR.

The contribution of this paper is to present the simultaneous optimal network reconfiguration and power allocation of distributed wind turbine using an improved genetic algorithm.

\section{DSR Formulation}

\subsection{Objective function}

The objective is to minimize the total network active loss which can be described as:

$$
\min P_{\text {loss }}=\sum_{i=1}^{N_{b r}} r_{i} \frac{P_{l i}^{2}+Q_{l i}^{2}}{V_{i}^{2}}
$$

Where, $P_{\text {loss }}$ is the system active power loss; $P_{l i}$ and $Q_{l i}$ are active and reactive power at the end terminal of branch $i$ respectively; $V_{i}$ is the end terminal voltage of branch $i ; r_{i}$ is the resistance of branch $i$.

\subsection{Constraints}

Network reconfiguration needs to satisfy the following constraints:

(1)Distribution power flow equations. For each bus $i$, the active and reactive power balance should be observed which can be expressed as: 


$$
\begin{aligned}
& P_{i}=\sum_{j=1}^{N_{b u s}}\left|V_{i}\right|\left|V_{j}\right|\left|Y_{i j}\right| \cos \left(\theta_{i j}-\delta_{i}+\delta_{j}\right) \\
& Q_{i}=\sum_{j=1}^{N_{b u s}}\left|V_{i}\right|\left|V_{j}\right|\left|Y_{i j}\right| \sin \left(\theta_{i j}-\delta_{i}+\delta_{j}\right)
\end{aligned}
$$

where, $\left|Y_{i j}\right|$ and $\theta_{i j}$ are the amplitude and angle of $i j$ entry of the bus admittance matrix; $V_{i}$ and $\delta_{i}$ are the amplitude and angle of bus $i$ respectively.

(2)Permissible range of bus voltage

$$
V_{\min } \leq\left|V_{i}\right| \leq V_{\max }
$$

where, $V_{\min }$ and $V_{\max }$ are the lower and upper limit of bus voltage.

(3)Branch power limits

$$
\sqrt{P_{l i}^{2}+Q_{l i}^{2}} \leq S_{i \max }
$$

where $S_{i \max }$ is the maximum power transmitted through branch $i$.

(4)Radial and connective network constraint. Distribution networks must have radial topologies with all of nodes are energized.

\section{Load flow with wind power generators}

\subsection{Model of wind power generators}

The bus that connected to a wind power generator is regarded as P-Q(V) bus, and for simplifying, the active power is regard as fixed value. The reactive power can be described as:

$$
Q=\frac{V^{2}}{X_{m}}+\frac{V^{2}-\sqrt{V^{4}-4 P^{2} X_{1}^{2}}}{2 X_{1}}
$$

where, $X_{1}$ and $X_{m}$ represent the leakage and excitation reactance of generator respectively.

Shunt capacitor is utilized to compensate for the reactive power that the wind power generators absorbed. Suppose the power factor of the wind turbines rises from $\cos \phi_{1}$ to $\cos \phi_{2}$, the reactive power that the shunt capacitors provides can be expressed as:

$$
\Delta Q_{c}=P_{e}\left(\sqrt{\frac{1}{\left(\cos \phi_{1}\right)^{2}}-1}-\sqrt{\frac{1}{\left(\cos \phi_{2}\right)^{2}}-1}\right)
$$

Then the number of the shunt capacitors that need to switch on is:

$$
n=\left[\frac{\Delta Q_{c}}{Q_{N-U n i t}}\right]
$$

Where, $Q_{N-\text { Unit }}$ represents the unit capacity of the shunt capacitors.

\subsection{Power flow calculation}

In order to get the power loss, the forward and backward sweep approach is applied to calculate the power flow of the distribution system with wind power generators. Fig. 1 shows the process.

\section{Reconfiguration based on genetic algorithm}

\subsection{Genetic encoding}

Here the decimal coding method is applied. The sequence of the branches is corresponding to a chromosome. The radial structure of network is obtained based on spinning tree theory to ensure all the solutions are feasible.

\subsection{Genetic decoding}

A spanning tree could be formed by genetic decoding. The steps to form a spanning tree are as follow:

(1) Put the branches that not take part in genetic coding into several subsets, which the connected branches are in the same subset. For a given distribution network, the number of the branches that not take part in genetic coding is fixed. 
(2) If the number of the branches in all the subsets equals the number of the tie switches, the procedure ends, otherwise the step (3) will be carried out.

(3) Put a new bus $B_{i}$ which corresponded with a gene of the chromosome into the existing subsets according to the following four situations.

(1) If no branch of the existing subsets connects to the new branch $B_{i}$, a new subset $T_{k}$ will be formed: $T_{k}=\left\{B_{i}\right\}$;

(2) If there is only one existing subset $T_{m}$ in which a branch connected to the new branch $B_{i}$, then join $B_{i}$ to subset $T_{m}$.

(3) If new branch $B_{i}$ connects to two existing subsets $T_{m}$ and $T_{n}, T_{m}$ and $T_{n}$ will merge into a new subset and the join branch $B_{i}$ to the new subset. All the branches of the new subset are connected each other.

(4) If there is one existing subset in which two branches are connected to the new branch $B_{i}$, then the branch $B_{i}$ will be neglected.

Go to step (2) to process other branches.

Fig. 2 shows the procedure of the proposed algorithm.

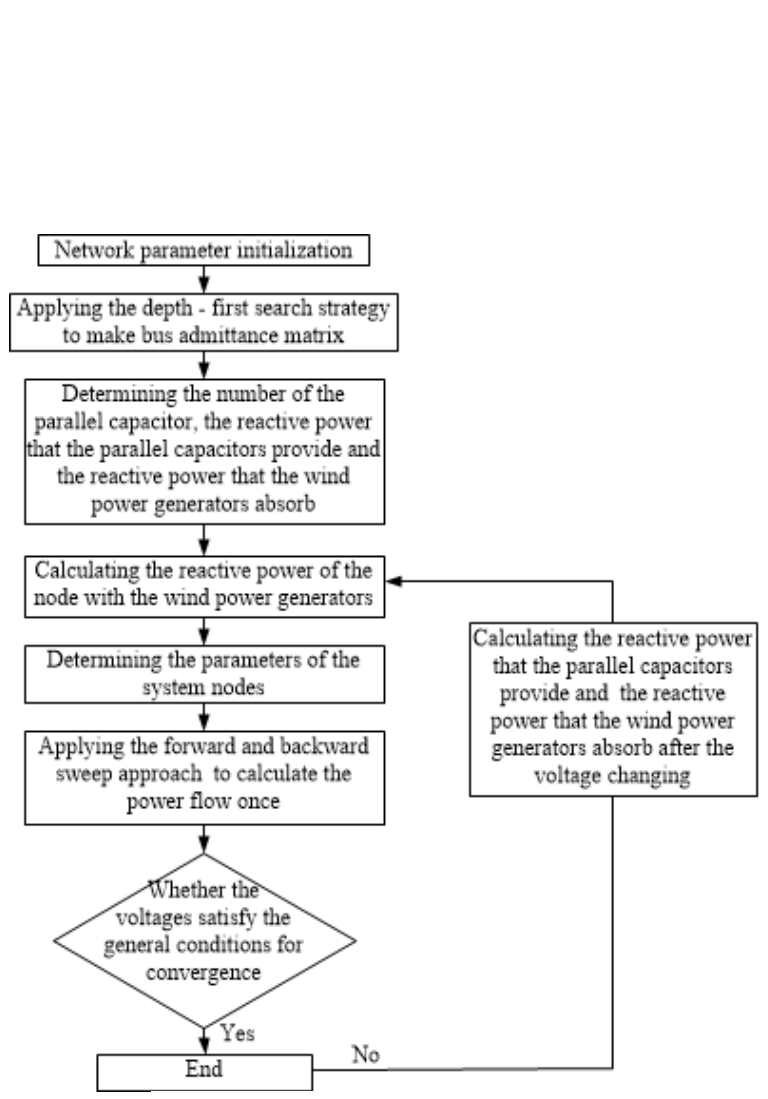

Fig.1 backward and forward sweep power flow

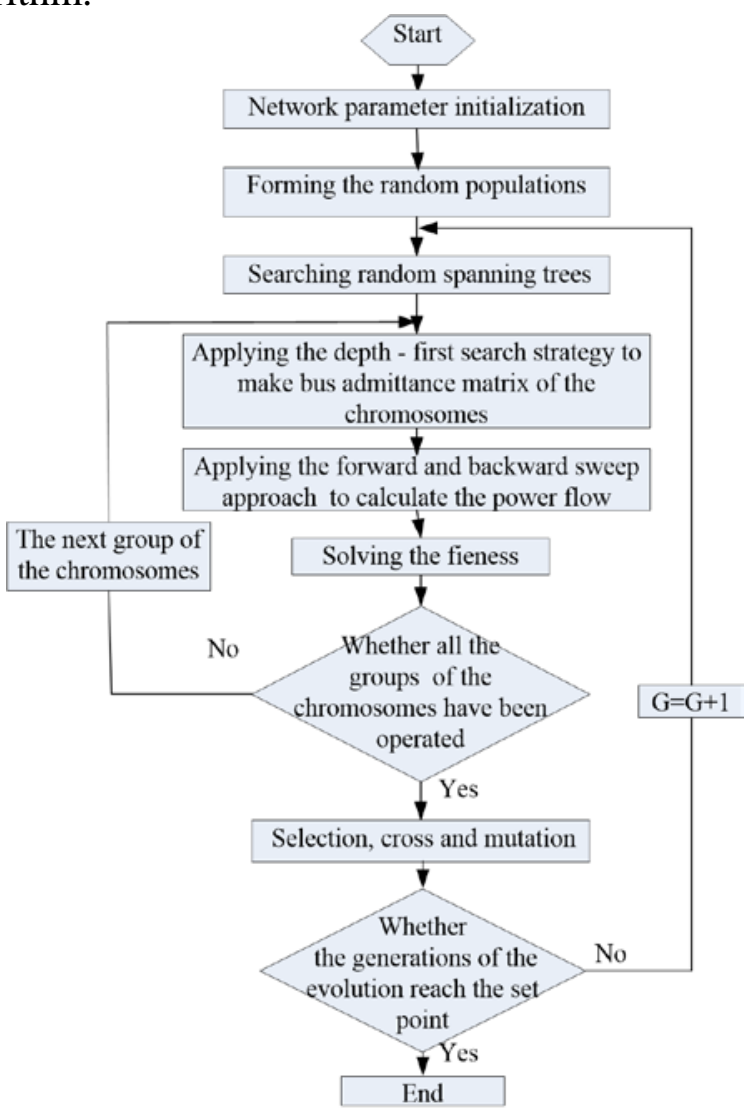

Fig.2 Flow chart of algorithm

\section{Simulation results}

Fig. 3 shows the typical IEEE-33 test system. The rated capacity of the wind turbine is $600 \mathrm{~kW}$. The unit capacity of the shunt capacitor is $10 \mathrm{kVar}$. The power factor of the wind turbine is 0.85 and is required to rise to 0.95 after switching the shunt capacitors. 


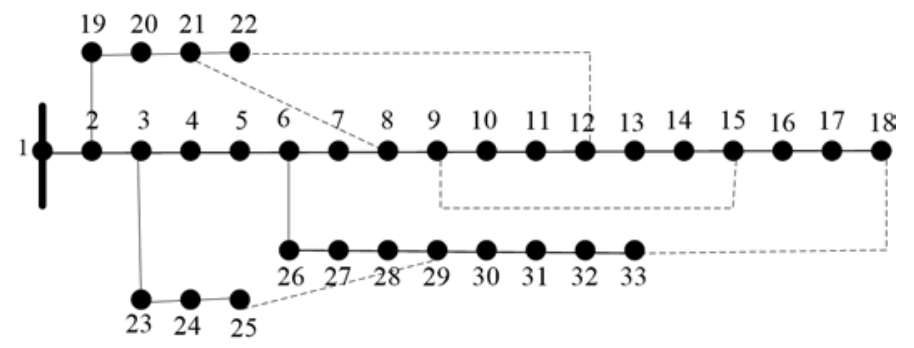

Fig.3 Schematic of 33 nodes system

Suppose the wind power generator is located at node 24. In order to investigate power loss and voltage quality of distribution system before and after reconfiguration, we change the active out power of the wind turbine from 0 to 300,350 , and $400 \mathrm{~kW}$.

Table 1 shows the scheme of the reconfiguration when the active powers of the wind power generator are different. The symbol $B_{a-b}$ represents the branch between the node $a$ and the node $b$.

Table1 Reconfiguration results of 33 nodes system

\begin{tabular}{cllll}
\hline $\begin{array}{c}\text { active power of wind } \\
\text { turbine }(\mathrm{kW})\end{array}$ & \multicolumn{1}{c}{ 0 } & 300 & 350 & 400 \\
\hline & $B_{7-8}$ & $B_{7-8}$ & $B_{7-8}$ & $B_{7-8}$ \\
opening & $B_{9-10}$ & $B_{9-10}$ & $B_{10-11}$ & $B_{11-12}$ \\
branches & $B_{14-15}$ & $B_{14-15}$ & $B_{14-15}$ & $B_{14-15}$ \\
& $B_{28-29}$ & $B_{28-29}$ & $B_{28-29}$ & $B_{28-29}$ \\
& $B_{32-33}$ & $B_{32-33}$ & $B_{32-33}$ & $B_{32-33}$
\end{tabular}

Table 2 shows the power loss and the lowest voltage of distribution system before and after reconfiguration when the active power of the wind power generator is $0,300,350,400 \mathrm{~kW}$.

Table2 Indices comparison before and after reconfiguration

\begin{tabular}{|c|c|c|c|c|c|c|}
\hline $\begin{array}{l}\text { active power of wind } \\
\text { turbine }(\mathrm{kW})\end{array}$ & \multicolumn{2}{|c|}{$\begin{array}{l}\text { lowest voltage } \\
\text { (p.u.) }\end{array}$} & $\begin{array}{l}\text { raised } \\
(\%)\end{array}$ & \multicolumn{2}{|c|}{ Power loss(kW) } & $\begin{array}{l}\text { decreased } \\
\text { (\%) }\end{array}$ \\
\hline \multirow{2}{*}{0} & Original & 0.9132 & \multirow{2}{*}{3.27} & Original & 202.68 & \multirow{2}{*}{31.15} \\
\hline & Optimized & 0.9413 & & Optimized & 139.54 & \\
\hline \multirow{2}{*}{300} & Original & 0.9143 & \multirow{2}{*}{3.29} & Original & 192.10 & \multirow{2}{*}{32.85} \\
\hline & Optimized & 0.9444 & & Optimized & 129.00 & \\
\hline \multirow{2}{*}{350} & Original & 0.9144 & \multirow{2}{*}{3.35} & Original & 190.75 & \multirow{2}{*}{32.76} \\
\hline & Optimized & 0.9450 & & Optimized & 128.26 & \\
\hline \multirow{2}{*}{400} & Original & 0.9146 & \multirow{2}{*}{3.38} & Original & 189.33 & \multirow{2}{*}{32.61} \\
\hline & Optimized & 0.9455 & & Optimized & 127.59 & \\
\hline
\end{tabular}

\section{Conclusions}

This paper proposed an approach to the distribution system reconfiguration in the presence of distributed generators. Simulation results show that the proposed method can reduce the power loss and improve the voltage quality of distribution system. On the conditions that the output of the wind power generators is less than the power load of the located node, the power loss becomes smaller and the voltage quality of distribution system becomes better when the active power of the wind turbine increases.

\section{Acknowledgment}

This work is supported by Shandong Science and Technology Development Project of China (2012G0020503) and Natural Science Foundation of Shandong Province, China (ZR2011FM008). 


\section{References}

[1] D. P. Bernardon, A. P. C. Mello, L. L. Pfitscher et al., Real-time reconfiguration of distribution network with distributed generation, Electric Power Systems Research, 107 (2014) 59-67.

[2] R. S. Rao, K. Ravindra, K. Satish et al., Power loss minimization in distribution system using network reconfiguration in the presence of distributed generation, IEEE Transactions on Power Systems, 28 (2013) 317-325.

[3] M. Sedighizadeh, M. Esmaili, and M. Esmaeili, Application of the hybrid Big Bang-Big Crunch algorithm to optimal reconfiguration and distributed generation power allocation in distribution systems, Energy, 76 (2014) 920-930

[4] A. M. Tahboub, V. R. Pandi, and H. H. Zeineldin, Distribution System Reconfiguration for Annual Energy Loss Reduction Considering Variable Distributed Generation Profiles, IEEE Transactions on Power Delivery, 4 (2015) 1677-1685.

[5] Abdelaziz AY, Mohamed FM, Mekhamer SF, Badr MAL. Distribution system reconfiguration using a modified Tabu Search algorithm. Electric Power Systems Research,8 (2010) 943-953.

[6] A.Y. Abdelaziz, F.M. Mohammed, S.F. Mekhamer, M.A.L. Badr, Distribution systems reconfiguration using a modified particle swarm optimization algorithm, International Journal of Electric Power Systems Research, 11(2009) 1521-1530. 